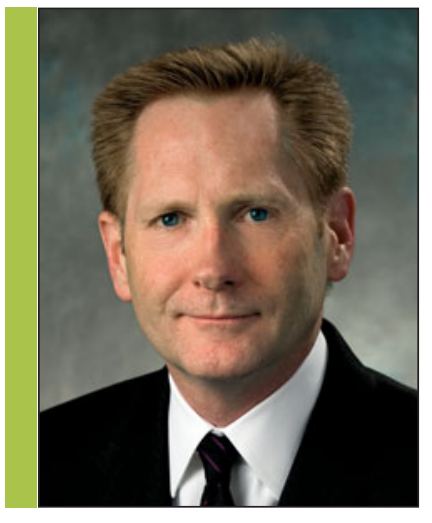

\title{
Eric J. Amis to give MRS plenary address
}

$\mathbf{E}$

ric J. Amis, director of Physical Sci-

ences at the United Technologies Research Center (UTRC), will deliver the plenary address at the 2011 Materials Research Society Fall Meeting. His presentation is titled "Three Materials, Three Challenges." The plenary session will be held in the Grand Ballroom of the Sheraton Hotel on Monday, Nov. 28 at 6:30 p.m.

Amis describes materials research as an enabler of technologies that address global needs. At UTRC, he considers the synergy between global technology needs and industry interests, concentrating on the areas of infrastructure, transportation, and energy. In his plenary presentation, Amis will consider examples of representative materials with tangible benefits and some challenges to enabling technologies within the DNA of corporate research.

Prior to joining UTRC in 2009, Amis spent 15 years in leadership roles at the National Institute of Standards and Technology (NIST) in the Materials Science and Engineering Laboratory, including 10 years in the Polymers Division. Be- fore joining NIST, he was a member of the chemistry faculty at the University of Southern California for 11 years. His $\mathrm{PhD}$ degree in chemistry is from the University of Wisconsin-Madison.

Amis is a fellow of the Materials Research Society, the American Physical Society (APS), and the Polymeric Materials: Science and Engineering Division of the American Chemical Society (ACS). He has served as chair of the Division of Polymer Physics of the APS and the Polymer Chemistry Division of the ACS, and he was editor-inchief of the Journal of Polymer

Science: Physics for 11 years. He also is past chair of the Versailles Project on Advanced Materials and Standards, and was vice president of the World Materials Research Institutes Forum. His research specialties are combinatorial and high-throughput methods for advanced materials, nanomaterials characterization, and soft matter physics. He has 150 peer-reviewed publications.

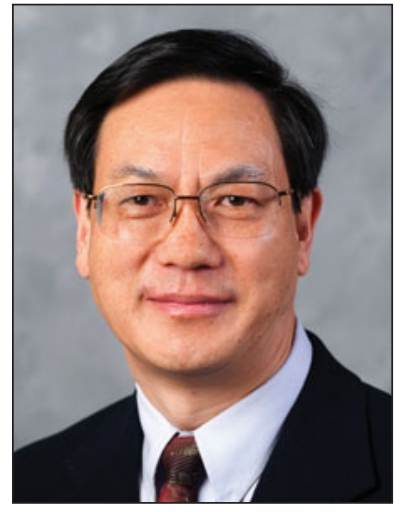

Zhong Lin Wang named 2011 MRS Medalist for oxide nanotechnology

T The Materials Research Society has named Zhong Lin (Z.L.) Wang of the Georgia Institute of Technology as MRS Medalist. He was cited for "seminal contributions in the discovery, controlled synthesis, and fundamental understanding of $\mathrm{ZnO}$ nanowires and nanobelts, and the design and fabrication of novel, nanowire-based nanosensors, piezotronic devices, and nanogenerators." Wang will be recognized during the awards ceremony at the 2011 MRS Fall Meeting in Boston. During the Meeting, he will present his award talk, "From nanogenerators to piezotronics - a decade study of $\mathrm{ZnO}$ nanostructures."

Wang has made seminal contributions to the discovery, controlled synthesis, understanding, and novel applications of $\mathrm{ZnO}$ nanowires and nanobelts in energy harvesting, as nanosensors, and in piezotronics. He is a pioneer in the invention and study of nanogenerators for selfpowered nanodevices and nanosystems, and he is responsible for breakthrough innovations for in situ mechanical and electrical property measurements of nanotubes in transmission electron microscopy, and in the fundamental understanding of the growth mechanisms of nanoparticles/nanowires and their selfassemblies. His work on nanogenerators and nanopiezotronics has opened a new field in nanotechnology, nanoscale energy generation, which has had a major impact on the development of nanodevices and self-powered nanosystems, with significant potential for biosensing, nanorobotics, microelectromechanical systems, defense technology, and consumer electronics.

Wang received his BS degree in applied physics from Xidian University (1982) and his $\mathrm{PhD}$ in physics from Arizona State University (1987). He has authored and co-authored four textbooks, edited 14 books, and published over 620 articles. He is a foreign member of the Chinese Academy of Sciences; a 